\title{
Mortality from respiratory diseases associated with opium use: a population-based cohort study
}

\author{
Atieh Rahmati, ${ }^{1}$ Ramin Shakeri, ${ }^{1}$ Hooman Khademii, ${ }^{1}$ Hossein Poutschi, ${ }^{1}$ \\ Akram Pourshams, ${ }^{1}$ Arash Etemadi, ${ }_{1}^{1,2}$ Masoud Khoshnia, ${ }^{3}$ Amir Ali Sohrabpour, ${ }^{1}$ \\ Ali Aliasgari, ${ }^{1}$ Elham Jafari, ${ }^{1}$ Farhad Islami, ${ }^{1,4}$ Shahryar Semnani, ${ }^{3}$ \\ Abdolsamad Gharravi, ${ }^{1,3}$ Christian C Abnet, ${ }^{2}$ Paul D P Pharoah, ${ }^{5}$ Paul Brennan, ${ }^{6}$ \\ Paolo Boffetta, ${ }^{7}$ Sanford M Dawsey, ${ }^{2}$ Reza Malekzadeh, ${ }^{1}$ Farin Kamangar ${ }^{1,8}$
}

\begin{abstract}
- Additional material is published online only. To view please visit the journal online (http://dx.doi.org/10.1136/ thoraxjnl-2015-208251).

For numbered affiliations see end of article.
\end{abstract}

\section{Correspondence to}

Professor Farin Kamangar, Department of Public Health Analysis, School of Community Health and Policy, Morgan State University, Portage Avenue Campus, Room 109, Baltimore, MD 21251, USA;

farin.kamangar@morgan.edu

$A R, R S, R M$ and FK contributed equally.

Received 2 January 2016 Revised 11 September 2016 Accepted 16 October 2016 Published Online First 24 November 2016

\section{CrossMark}

To cite: Rahmati $\mathrm{A}$,

Shakeri R, Khademi $\mathrm{H}$, et al.

Thorax 2017;72:1028-1034.

\begin{abstract}
Background Recent studies have suggested that opium use may increase mortality from cancer and cardiovascular diseases. However, no comprehensive study of opium use and mortality from respiratory diseases has been published. We aimed to study the association between opium use and mortality from respiratory disease using prospectively collected data. Methods We used data from the Golestan Cohort Study, a prospective cohort study in northeastern Iran, with detailed, validated data on opium use and several other exposures. A total of 50045 adults were enrolled from 2004 to 2008, and followed annually until June 2015 , with a follow-up success rate of $99 \%$. We used Cox proportional hazard regression models to evaluate the association between opium use and outcomes of interest.
\end{abstract}

Results During the follow-up period, 331 deaths from respiratory disease were reported ( 85 due to respiratory malignancies and 246 due to non-malignant aetiologies). Opium use was associated with an increased risk of death from any respiratory disease (adjusted $\mathrm{HR} 95 \% \mathrm{Cl} 3.13$ (2.42 to 4.04)). The association was dose-dependent with a HR of 3.84 (2.61 to 5.67) for the highest quintile of cumulative opium use versus never use $\left(P_{\text {trend }}<0.001\right)$. The HRs $(95 \% \mathrm{Cl})$ for the associations between opium use and malignant and non-malignant causes of respiratory mortality were 1.96 (1.18 to 3.25$)$ and 3.71 (2.76 to 4.96), respectively.

Conclusions Long-term opium use is associated with increased mortality from both malignant and nonmalignant respiratory diseases.

\section{INTRODUCTION}

The United Nations Office of Drugs and Crime estimates that 16.5 million people use opium or its derivatives illicitly. ${ }^{1}$ The acute effects of opium use - such as its analgesic and soporific effects-have been known for millennia. However, relatively few well-designed human or animal studies have investigated the long-term effects of opium use on body organs, including organs of the respiratory tract. As a result, the current understanding of long-term effects of opium use is not based on solid scientific findings. A popular, yet unsubstantiated, belief among lay people and some of the older generation of physicians in Central Asia is that long-term use
Key messages

What is the key question?

- What are the effects of chronic opium use on mortality due to malignant and non-malignant respiratory disease?

What is the bottom line?

- Long-term opium use is associated with increased mortality from both malignant and non-malignant respiratory diseases.

\section{Why read on?}

- The results of this study shed light on a relatively new risk factor for death from respiratory diseases. The results are relevant to both clinicians and public health practitioners.

of low-dose opium can increase longevity by preventing chronic diseases. This belief has been challenged by clinical and epidemiological studies showing otherwise.

Reports of lung injury due to opium abuse date back to the 1880s, when Sir William Osler mentioned lung oedema as a result of morphine poisoning. ${ }^{2}$ In 1930, Gaide and Neuberger reported a higher frequency of productive cough and dyspnoea in opium smokers. ${ }^{3}$ Other researchers have also reported a higher frequency of cough, radiological abnormalities and fibrosis in opium users. ${ }^{4-6}$ For example, studying 48 opium users with a $\mathrm{FEV}_{1}$ of $<60 \%$, Da Costa et al reported high rates of bronchitis and bronchiolitis, destructive emphysema and peribronchiolar fibrosis. ${ }^{4}$ However, these clinical studies were limited by small sample sizes, lack of control groups and possible confounding by cigarette smoking and other factors. More recently, epidemiological studies of laryngeal cancer, ${ }^{7}$ and lung cancer ${ }^{9}{ }^{10}$ in relation to opium use have been conducted, which have controlled for smoking. However, these studies have had retrospective casecontrol designs, which may be subject to control selection bias, recall bias and interviewer bias. Prospective cohort studies may provide more valid information on the chronic effects of opium use, yet data from such studies are limited. 
We used data from the Golestan Cohort Study (GCS), a large prospective cohort study of over 50000 individuals in northeastern Iran, to examine the association of opium use and subsequent mortality from both malignant and non-malignant respiratory diseases.

\section{METHODS}

\section{Study population}

The GCS is a prospective cohort study of 50045 adults, aged 40-75 years, from Golestan Province in northeastern Iran. A detailed description of the study population, its methods and follow-up is available elsewhere. ${ }^{11}{ }^{12}$ In brief, a total of 10033 people from Gonbad City, the largest city in the study area, and 40012 people from 326 villages in the area were enrolled between January 2004 and June 2008. The study population is a sample of the Golestan province population, aged 4075 years. The primary goal was to launch a cohort of 50000 healthy individuals, with equal numbers of men and women, $20 \%$ from urban areas and $80 \%$ of Turkmen ethnicity. A total of 16599 urban dwellers older than 40 years were selected randomly from five areas of Gonbad City by systematic clustering based on household number. The selected inhabitants were contacted at home by specially trained health workers and invited to visit the GCS Center. In rural areas, all residents of all villages in the study catchment area who were eligible for this study were invited to participate. At the end of enrolment, compared with participants, the non-participants were more likely to be men and to live in urban areas. The distribution of participants by place of residence and ethnicity is close to the initial goal; however, possibly because of lower employment rate, participation rates in women were higher than that of men $(70 \%$ vs $50 \%)$. The design and conduct of the study were approved by the ethical review boards of Digestive Disease Research Center of Tehran University of Medical Sciences, the International Agency for Research on Cancer and the US National Cancer Institute, and all participants signed a written informed consent at enrolment.

\section{Exposure and other covariate assessment}

Opium use was assessed using a structured questionnaire, which included detailed questions about the age of starting opium use, daily amount and frequency of use (how many days a week if weekly or more), routes of administration, opium types and the age of quitting for those who had quit. Routes of administration included opium smoking, ingestion or both. Opium types included teriak, sukhteh and shireh. Teriak is a sticky paste prepared by boiling raw opium for a few hours. Sukhteh is the dry, black residue of smoked teriak, which sticks to the opium pipe. It can be scraped from the opium pipe and ingested. Shireh is a refined opium product often made by boiling a combination of opium and sukhteh in hot water and passing the solution through filters several times. Heroin is also obtained from opium, but its use among the cohort participants was rare, so it was not a category evaluated in this study. If subjects used several types of opium, or they used opium intermittently (stopped and resumed use), data were recorded separately for each type and period of use. The accuracy of self-reported opium use in this population was evaluated by comparing selfreported opium use with the presence of codeine or morphine in urine samples of 150 cohort participants. That study showed a high accuracy for self-report of opium use in these cohort participants, with a sensitivity of 0.93 and a specificity of $0.89 .{ }^{13}$

Other potential disease determinants in the study, including age, gender, place of residence, ethnicity, marital status, tobacco use, alcohol drinking and education were also assessed using a structures questionnaire. Place of residence was categorised as rural or urban. Ethnic background was divided into Turkmen (the main ethnic group in the area) and non-Turkmen. Tobacco and alcohol use questions included details similar to those for opium use, that is, age of starting use, daily amount and frequency of use, types of tobacco and the age of quitting consumption, if the person had quit. The main types of tobacco use in the area were cigarettes, hookah (waterpipe) and nass. Nass is a chewing tobacco product, which is a combination of tobacco, ash and lime. Alcohol was used infrequently and in small amounts.

\section{Cause of death ascertainment}

The follow-up methods and cause of death ascertainment in the GCS have been described in detail elsewhere. ${ }^{11}$ The follow-up team uses several methods to reach the study participants or to collect information on the cause of death. These methods include annual phone calls to study participants or their relatives, communicating with local health workers and collecting data from local death registries and cancer registries. All cohort participants are phoned annually and asked about vital status and any admission to a hospital or outpatient clinic, and the information is recorded. If the participant is not reached after seven phone calls made in a 2 -week period, friends or local health workers are contacted. Using these methods, the follow-up success rate so far is over $99 \%$.

If a death is reported, the physician in the follow-up team uses a validated verbal autopsy questionnaire to interview the closest relative of the deceased. ${ }^{12}$ In addition, the follow-up team actively pursues and collects all available medical documents such as hospital charts, physician notes, ECG reports and pathology reports from all medical centres in Golestan Province and the neighbouring provinces. Two internists independently review all collected documents to make a diagnosis according to the International Statistical Classification of Diseases-10 coding system. If the two diagnoses are concordant, the diagnosis is made. If not, a third more experienced internist reviews all documents and the two initial diagnoses to make the final diagnosis. If a final diagnosis cannot be made for any reason, the cause of death is coded as 'unknown'. For the purpose of this paper, we focused on the main respiratory causes of death, both malignant and non-malignant. The major malignant causes of death were lung cancer and laryngeal cancer. The main causes of non-malignant death were COPD, asthma and pneumonia.

\section{Statistical analysis}

Age-standardised mortality rates were calculated using the World Population 2000-2025 as the reference population.

We used Cox proportional hazards regression models, with age as the timescale, to estimate HRs and 95\% CIs for mortality due to respiratory diseases in association with opium use. The proportional hazard assumption was verified using Schoenfeld residuals. Follow-up continued until death, loss to follow-up or 10 July 2015, whichever came first. The covariates in the adjusted models included age, sex, place or residence (rural or urban), education, marital status and any tobacco use (including cigarette smoking, hookah use or chewing nass).

Ever opium users were defined as those participants who had ever used opium at least once a week for at least 6 months. Former opium users were defined as participants who had reported quitting their habit at least 1 year before the enrolment. For current users, duration of use and cumulative use (defined as duration of use, in days, multiplied by daily amount 
of opium use, in nokhods, a local unit for opium consumption that weighs about $0.2 \mathrm{~g}$ ) were categorised into quintiles to test for dose-response relationships. Data were analysed by routes of administration and types of opium used. In addition, the results are presented for death from each major type of malignant (lung cancer and laryngeal cancer) and non-malignant (COPD, asthma and pneumonia) outcomes. To address reverse causality, we conducted a sensitivity analysis excluding subjects who had used opium for $<10$ years, and also excluding deaths occurring in the first 24 months of follow-up.

Statistical analyses were conducted with STATA statistical software, V.12 (STATA, College Station, Texas, USA). All p values are two-sided, and $\mathrm{p}$ values $<0.05$ and $95 \%$ CIs not including one were considered as statistically significant.

\section{RESULTS}

During the follow-up period, 331 deaths from respiratory diseases were reported, including 85 from malignant and 246 from non-malignant diseases.

Table 1 shows the distribution of demographic factors, education and habits including alcohol consumption, cigarette smoking, in GCS, opium users and non-users. In all, 8487 of the 50045 cohort participants (17\%) of the entire cohort ever used opium. The main factors associated with higher percentage of opium use included cigarette smoking, alcohol consumption, being male, older ages, Turkmen ethnicity and rural residency.

The association of opium use with respiratory mortality is further explored in table 2. After adjusting for age, sex, residential place, education, marital status, drinking alcohol and cumulative use of any kind of tobacco (pack-years for cigarette smoking and amount $\times$ duration of use for hookah and nass), opium consumption was associated with a statistically significant higher risk of all respiratory mortality, with an $\mathrm{HR}(95 \% \mathrm{CI})$ of 3.13 (2.42 to 4.04). The corresponding HRs (95\% CI) for death from malignant and non-malignant causes were 1.96 (1.18 to 3.25 ) and 3.71 (2.76 to 4.96$)$. When the results were divided by quintiles of duration of use or cumulative use in the current users, there were dose-response increases, with the highest HRs in the fifth quintiles and $p$ values for trend of $<0.001$. Increased risks were seen for the two main types of opium products used in the area (ie, teriak and shireh), with combination use having the highest HR. Likewise, increased risks were observed for both opium smoking and ingestion, and the highest HRs were in those who both smoked and ingested opium.

Table 3 shows opium data, stratified by type of malignancy and non-malignant diseases. Point estimates showed increased risk of lung cancer, laryngeal cancer, COPD, asthma and pneumonia associated with opium use. The results were not statistically significant for lung cancer and laryngeal cancer, but very close to significance, with lower bounds of CIs being 0.99 for both cancers. Table 3 also shows the results of sensitivity analysis excluding deaths that occurred during the first 24 months of follow-up, and also results of an analysis excluding the 3952 subjects who had used opium for $<10$ years. The results did not change materially after excluding deaths in the first 24 months, but the HRs were slightly stronger after excluding those who had used opium for $<10$ years.

Although we have adjusted for tobacco use, since there is a possibility that some of the increased mortality be driven from residual confounding from tobacco use or its interaction effect with opium, we performed a sensitivity analysis restricted to never smokers to assess the effect of tobacco use.

\section{DISCUSSION}

In Central Asia, some lay people and even some older physicians believe that opium use increases longevity. The reason may be pain-relieving effects of opium use, which may be misinterpreted as curative effects. However, the results of our study show that opium use is associated with increased mortality from respiratory diseases. These results add to other recent literature that opium is indeed associated with higher risk of both malignant and non-malignant diseases.

With regard to malignant diseases, a case series published in 1968 was perhaps the first study that suggested that opium may be a carcinogen, ${ }^{14}$ showing potential malignant effects of opium on tongues of the users. Whereas a few other studies evaluating the carcinogenicity of opium appeared in the literature in the 1970s and early 1980s, there was a hiatus for nearly two decades, and most studies of opium and cancer have been published in the last 12 years. ${ }^{15}$ Epidemiological studies have associated opium use with increased risk of cancers of the oral

Table 1 Descriptive characteristics of the cohort participants, opium users and non-users

\begin{tabular}{|c|c|c|c|c|c|}
\hline & Never opium user, $\mathbf{n}(\%)$ & Ever opium user, $n(\%)$ & & Never opium user, $\mathbf{n}(\%)$ & Ever opium user, $\mathrm{n}(\%)$ \\
\hline All cohort participants & $41558(83)$ & 8487 (17) & & & \\
\hline Age categories (years) & & & Education & & \\
\hline $30-39$ & $306(94)$ & $19(6)$ & Illiterate & $29490(84)$ & $5628(16)$ \\
\hline $40-49$ & $19462(85)$ & $3331(15)$ & Up to 8 years & $8480(79)$ & $2228(21)$ \\
\hline $50-59$ & $13287(82)$ & 2845 (18) & High school & $2601(82)$ & $554(18)$ \\
\hline $60-69$ & $6336(79)$ & $1641(21)$ & Higher education & $987(93)$ & $77(7)$ \\
\hline 70 or more & $2167(77)$ & $651(23)$ & & & \\
\hline Gender & & & Ethnicity & & \\
\hline Female & $26456(92)$ & $2355(8)$ & Non-Turkmen & $10857(85)$ & $1935(15)$ \\
\hline Male & $15102(71)$ & $6132(29)$ & Turkmen & 30701 (82) & $6552(18)$ \\
\hline Place of residence & & & Marital status & & \\
\hline Rural & 32527 (81) & 7485 (19) & Married & $36345(83)$ & 7545 (17) \\
\hline Urban & $9031(90)$ & $1002(10)$ & Widow/widower, single/divorce & $5185(85)$ & $940(15)$ \\
\hline Alcohol consumption & & & Cigarette smoking & & \\
\hline Never use & $40754(84)$ & $7562(16)$ & Never smokers & 36255 (93) & $2933(7)$ \\
\hline Ever use & $804(47)$ & $925(54)$ & Ever smokers & $5303(49)$ & $5554(51)$ \\
\hline
\end{tabular}


Table 2 HRs for the association between opium use and respiratory disease mortality by type of opiate used, route of administration and cumulative use

\begin{tabular}{|c|c|c|c|c|c|c|}
\hline & \multicolumn{2}{|l|}{ All respiratory diseases } & \multicolumn{2}{|l|}{ Malignant respiratory diseases } & \multicolumn{2}{|c|}{ Non-malignant respiratory diseases } \\
\hline & No. of participants (deaths) & Cox HR* $(95 \% \mathrm{Cl})$ & No. of participants (deaths) & Cox HR $(95 \% \mathrm{Cl})$ & No. of participants (deaths) & Cox HR $(95 \%$ Cl) \\
\hline \multicolumn{7}{|l|}{ Opium use } \\
\hline Never users & 41558 (164) & Reference & $41558(42)$ & Reference & 41558 (122) & Reference \\
\hline Ever users & 8487 (167) & 3.13 (2.42 to 4.04$)$ & 8487 (43) & 1.96 (1.18 to 3.25$)$ & 8487 (124) & 3.71 (2.76 to 4.96 ) \\
\hline \multicolumn{7}{|l|}{ Opium use } \\
\hline Never users & 41558 (164) & Reference & $41558(42)$ & Reference & 41558 (122) & Reference \\
\hline Former users & $838(19)$ & 2.85 (1.73 to 4.7$)$ & $838(5)$ & 1.95 (0.73 to 5.16$)$ & $838(14)$ & 3.29 (1.84 to 5.88$)$ \\
\hline Currentt users & 7107 (143) & 3.31 (2.53 to 4.32 ) & 7107 (38) & 2.11 (1.25 to 3.55$)$ & $7107(105)$ & 3.92 (2.88 to 5.32 ) \\
\hline \multicolumn{7}{|l|}{ Duration of opium use } \\
\hline Never users & 41558 (164) & Reference & $41558(42)$ & Reference & 41558 (122) & Reference \\
\hline Former users & $868(20)$ & 3.03 (1.86 to 4.95$)$ & $868(5)$ & 2.01 (0.75 to 5.31 ) & $868(15)$ & 3.53 (2.01 to 6.2$)$ \\
\hline 1st quintile ( $\leq 3$ years) & $1671(17)$ & 2.19 (1.32 to 3.64$)$ & $1671(3)$ & 1.11 (0.34 to 3.66$)$ & $1671(14)$ & 2.68 (1.53 to 4.71$)$ \\
\hline 2nd quintile (4-7 years) & $1486(16)$ & 2.17 (1.28 to 3.66$)$ & $1486(2)$ & 0.73 (0.17 to 3.08 ) & $1486(14)$ & 2.87 (1.63 to 5.06$)$ \\
\hline 3rd quintile (8-12 years) & $1433(25)$ & 3.78 (2.43 to 5.89$)$ & $1433(5)$ & 1.77 (0.67 to 4.66$)$ & $1433(20)$ & 4.84 (2.94 to 7.94$)$ \\
\hline 4th quintile (13-20 years) & $1577(33)$ & 3.61 (2.41 to 5.4$)$ & $1577(10)$ & 2.58 (1.22 to 5.44$)$ & $1577(23)$ & 4.04 (2.51 to 6.5$)$ \\
\hline \multirow[t]{2}{*}{ 5th quintile (>20 years) } & $1452(56)$ & 4.14 (2.89 to 5.94$)$ & $1452(18)$ & 3.01 (1.55 to 5.81 ) & $1452(38)$ & 4.66 (3.04 to 7.15$)$ \\
\hline & & $p$ for trend $<0.000$ & & $p$ for trend $<0.001$ & & $p$ for trend $<0.000$ \\
\hline \multicolumn{7}{|l|}{ Opium cumulative use } \\
\hline Never users & $41558(164)$ & Reference & $41558(42)$ & Reference & 41558 (122) & Reference \\
\hline Former users & $868(20)$ & 2.99 (1.84 to 4.88$)$ & $868(5)$ & 1.99 (0.75 to 5.27$)$ & $868(15)$ & $3.47(1.98$ to 6.1$)$ \\
\hline 1st quintile ( $\leq 1148)$ & $1524(18)$ & 2.27 (1.38 to 3.73$)$ & $1524(2)$ & 0.73 (0.17 to 3.09 ) & $1524(16)$ & $3(1.76$ to 5.11$)$ \\
\hline 2nd quintile (1149 to 4383 ) & $1524(22)$ & 2.75 (1.74 to 4.36$)$ & $1524(5)$ & 1.64 (0.63 to 4.28$)$ & $1524(17)$ & 3.27 (1.94 to 5.51$)$ \\
\hline 3rd quintile (4384 to 12 054)) & $1524(25)$ & 3.03 (1.95 to 4.71$)$ & $1524(6)$ & 1.92 (0.78 to 4.68 ) & $1524(19)$ & 3.55 (2.15 to 5.88$)$ \\
\hline 4th quintile (12 055 to 30681 ) & $1570(38)$ & 4.15 (2.82 to 6.08$)$ & $1570(9)$ & 2.38 (1.09 to 5.18 ) & $1570(29)$ & 5.05 (3.26 to 7.83 ) \\
\hline \multirow[t]{2}{*}{ 5th quintile (>30 682) } & $1477(44)$ & 3.84 (2.61 to 5.67 ) & $1477(16)$ & 2.95 (1.48 to 5.88 ) & $1477(28)$ & 4.13 (2.57 to 6.64 ) \\
\hline & & $p$ for trend $<0.000$ & & $p$ for trend $<0.001$ & & $p$ for trend $<0.000$ \\
\hline \multicolumn{7}{|l|}{ Type of opium product } \\
\hline Never users & $41558(164)$ & Reference & $41558(42)$ & Reference & 41558 (122) & Reference \\
\hline Teriak only & $7308(142)$ & 3.11 (2.39 to 4.04$)$ & $7308(37)$ & 2.01 (1.19 to 3.35 ) & $7308(105)$ & 3.63 (2.69 to 4.9 ) \\
\hline Shireh only & $781(13)$ & 2.91 (1.62 to 5.21 ) & $781(2)$ & 1.06 (0.25 to 4.53$)$ & $781(11)$ & 3.99 (2.11 to 7.56$)$ \\
\hline Combinations & $398(12)$ & 4.31 (2.31 to 8.01 ) & $398(4)$ & 3.06 (1.02 to 9.18$)$ & $398(8)$ & 4.88 (2.31 to 10.37$)$ \\
\hline \multicolumn{7}{|l|}{ Route of administration } \\
\hline Never users & 41558 (164) & Reference & $41558(42)$ & Reference & 41558 (122) & Reference \\
\hline Smoking & $5804(66)$ & 2.21 (1.61 to 3.01 ) & $5804(21)$ & 1.69 (0.94 to 3.03$)$ & $5804(45)$ & 2.39 (1.65 to 3.45$)$ \\
\hline Ingestion & $2182(79)$ & 4.57 (3.35 to 6.23$)$ & $2186(17)$ & 2.29 (1.21 to 4.36$)$ & $2186(63)$ & 5.79 (4.08 to 8.21$)$ \\
\hline Both methods & $497(21)$ & 5.65 (3.45 to 9.25$)$ & $497(5)$ & 2.99 (1.11 to 8.06$)$ & $497(16)$ & 7.17 (4.07 to 12.62 ) \\
\hline
\end{tabular}




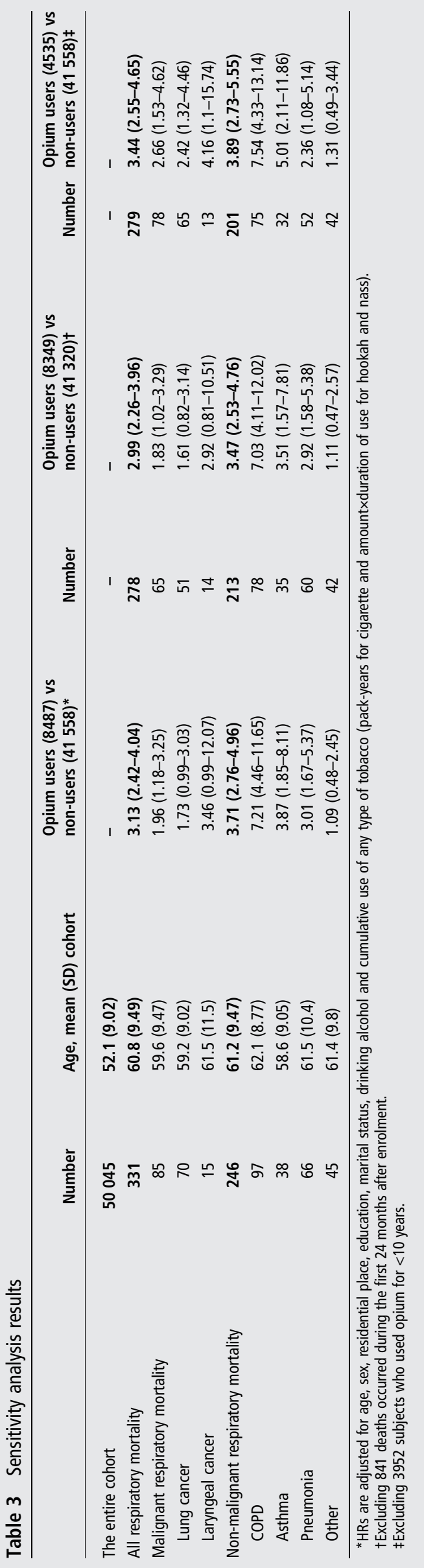

cavity, ${ }^{14} 16$ larynx, ${ }^{7} 8$ lung, ${ }^{9}{ }^{10}$ oesophagus, ${ }^{17-20}$ stomach $^{20-23}$ and bladder. ${ }^{24-29}$ The two previous epidemiological studies of opium in relation to laryngeal cancer ${ }^{78}$ had a retrospective casecontrol design and showed statistically significant increased risks, with ORs close to 10 . Previous studies ${ }^{10}$ have also shown an increased risk of lung cancer, with relative risks of close to 2 or 3 in opium users. Our study results did not show statistically significant increased risks of lung cancer or laryngeal cancer in opium users, but the point estimates of the HRs were above 1 and consistent with the findings of the previous studies. Therefore, lack of significance may be due to relatively small numbers of death (especially from laryngeal cancer) and hence low statistical power.

The epidemiological findings showing increased risk of several cancers with opium use are supported by laboratory studies showing that, after metabolic activation, opium dross has mutagenic activity in Salmonella typhimurium strains TA98 and TA $100 ;{ }^{30}$ causes frameshift mutations in S. typhimurium strains TA1538 and TA98 ${ }^{29}$ and induces sister chromatid exchanges. ${ }^{31}$ These effects are mostly attributed to nitrogen-containing heterocyclic compounds derived from pyrolysis of morphine. ${ }^{32}$ Studies have also shown that opium use may be associated with higher risk of other chronic diseases, such as cardiovascular diseases. ${ }^{33}$

There is also some relatively old literature on the effects of opium on non-malignant diseases of the lungs and pleura. ${ }^{3-6}$ Whereas these studies were case series, and not formally controlled for smoking cigarettes, they still provide important insights. For example, comparing studies of opium users (nearly all of whom also smoked cigarettes) with those who only smoked cigarette (and not opium), Da Costa et al found that the prevalence of parenchymal and pleural fibrosis was much higher in those who used opium. ${ }^{4}$ In addition, there were distinctive radiographic features-described as ill-defined nodular shadows with or without reticular patterns-in opium users that were not seen in pure cigarette smokers. ${ }^{4}$ More recent study from the UK has also shown the association between heroin inhalation and early onset emphysema. ${ }^{34}$ Our results are consistent with the findings of these clinical studies, and suggest an increased risk of death from asthma, COPD and pneumonia associated with opium use.

Cigarette smoking increases the risk of lung cancer in this population, as it does in other populations. However, for reasons that are not completely understood, probably due to the presence of other strong risk factors, ${ }^{35}$ lower overall amount of smoking or starting smoking later in life, tobacco smoking in Iran is a much weaker risk factor for total mortality, lung cancer and other diseases, than what is reported elsewhere. This has been shown repeatedly in studies in the 1970s, as well as in more recent studies. In the GCS, for example, the adjusted HR for opium and mortality was 1.86 , whereas the adjusted for smoking and mortality was $<1.30 .{ }^{36}$ In case-control studies in the $1970 \mathrm{~s},{ }^{37}$ as well as in more recent case-control studies, tobacco smoking increased the risk of oesophageal squamous cell carcinom (OSCC) less than twofold, ${ }^{17}$ was not associated with increased risk of gastric cancer ${ }^{38}$ and was not associated with risk of pancreatic cancer. ${ }^{39}$ We also see a relatively weak HR for lung cancer. Therefore, cigarette smoking cannot be a major source of confounding.

For a factor to be a major confounder, it has to be a strong risk factor of the outcome, so we believe that tobacco smoking is not major confounder but may interact with opium in causing cancer. In this study, using opium plus smoking increased the risk of cancer far beyond smoking alone, whereas opium uses alone did not increase risk of cancer. This finding, the points 
discussed above and results of other studies of opium and cancer collectively suggest an interaction as the most plausible explanation. However, there may be other alternative explanations too.

The strengths of our study include its prospective design, large sample size, extensive data collection for the exposure of interest (opium) and potential confounders (eg, cigarette smoking, age and sex) and conducting sensitivity analyses. In order to decrease the possibility of bias, the medical doctors who determined the causes of death were unaware of the history of opium use by the deceased (blind evaluation).

However, the study has limitations too. Although the accuracy of self-reported opium use in this population is high, some degree of misclassification due to recall bias is possible. On the other hand, there may be also some degrees of misclassification of cause of death, in spite of the validity of the verbal autopsy. Despite conducting a sensitivity analysis, reverse causality cannot be ruled out. COPD, asthma and laryngeal cancer are all associated with chronic coughs, and opium is a well-known antitussive. Therefore, it is possible that those who developed these diseases in Golestan Province used opium to suppress their cough, and excluding only 1 year may not be adequate to capture that. Also, small numbers of deaths in some subgroups (eg, laryngeal cancer) may result in low statistical power. Relatively little mechanistic studies have been done on the effects of chronic use of opium, so further research is needed to elucidate the mechanisms and check the plausibility of the findings.

In conclusion, our study showed an association between longterm opium use and higher mortality from both malignant and non-malignant respiratory causes. This study adds to the current literature showing detrimental health effects of long-term opium use.

\footnotetext{
Author affiliations

${ }^{1}$ Digestive Oncology Research Center, Digestive Disease Research Institute, Shariati Hospital, Tehran University of Medical Sciences, Tehran, Iran

${ }^{2}$ Division of Cancer Epidemiology and Genetics, National Cancer Institute, Bethesda, Maryland, USA

${ }^{3}$ Golestan Research Center of Gastroenterology\& Hepatology (GRCGH), Golestan

University of Medical Sciences, Gorgan, Iran

${ }^{4}$ American Cancer Society, Atlanta, Georgia, USA

${ }^{5}$ Departments of Oncology and Public Health and Primary Care University of

Cambridge, Cambridge, UK

${ }^{6}$ International Agency for Research on Cancer, Lyon, France

${ }^{7}$ The Tisch Cancer Institute and Institute for Translational Epidemiology, Icahn School of Medicine at Mount Sinai, New York, New York, USA

${ }^{8}$ Department of Public Health Analysis, School of Community Health and Policy, Morgan State University, Baltimore, Maryland, USA
}

Acknowledgements We thank the study participants for their cooperation over many years; the behvarz (community health workers) in the study areas for their help; Goharshad Goglani, Karim Aghcheli, Mehdi Nouraei, Akbar Fazeltabar Malekshah, Nasser Rakhshani, Masoud Sotoudeh, Ali Yoonessi, Mohsen Sadatsafavi, Alireza Sadjadi, Amir Sharifi and Mohammad R Akbari from DDRC. We also thank the general physicians, nurses and nutritionists in the enrolment teams for their collaboration and assistance and Golestan University of Medical Sciences (Gorgan, Iran), the Golestan health deputies and the chiefs of the Gonbad and Kalaleh health districts for their close collaboration and support.

Contributors Conception or design of the work: AR, RS, HK, RM, FK, PB, SMD, CCA, PDPP and PBr. The acquisition, analysisor interpretation of data for the work: $A R, R S, H K, H P, A E, M K, A A S, A A, E J, F I, S S, S G$ and FK. Drafting the manuscript for important intellectual content: AR, RS, FK, RM, FI, PB, CCA and SMD. Taking responsibility for the integrity of the work as a whole, from inception to published article: FK, RM, PB, SMD and CCA.

Funding The Golestan Cohort Study was supported by Tehran University of Medical Sciences (grant no: 81/15), Cancer Research UK (grant no: C20/A5860), Intramural Research Program of the US National Cancer Institute, National Institutes of Health and through various collaborative research agreements with the International Agency for Research on Cancer. The study has also received special support from the Social Security Organisation of Iran Golestan Branch.
Competing interests None declared.

Patient consent Obtained.

Ethics approval Ethical review boards of Digestive Disease Research Center of Tehran University of Medical Sciences, the International Agency for Research on Cancer and the US National Cancer Institute.

Provenance and peer review Not commissioned; externally peer reviewed.

\section{REFERENCES}

1 UNODC. World Drug Report_2011: United Nations Office on Drugs and Crime, 2011. http://www.unodc.org/documents/data-and-analysis/WDR2011/The opium-heroin_market.pdf

2 Osler W. Oedema of left lung-morphia poisoning. Montreal Gen Hosp Rep 1880;1:291.

3 Gaide LJ, Neuberger L. Le visage inconnu de l'opium: Impr. d'Extrême-Orient, 1930.

4 Da Costa JL, Tock EP, Boey HK. Lung disease with chronic obstruction in opium smokers in Singapore. Clinical, electrocardiographic, radiological, functional and pathological features. Thorax 1971;26:555-71.

5 Mazaud R, Andre L, Cuu L, et al. Le poumon des fumeurs dopium. Presse Medicale 1963;71:1800.

6 Khoo F, HENO TC, Koon LH. An unusual form of reticulated lung disease, probably an unidentified type of pneumoconiosis. A preliminary report. Singapore Med J 1960;1:96-8

7 Khoo R. Radiotherapy of carcinoma of the larynx. Ann Acad Med Singapore 1981:10:307-10.

8 Mousavi MR, Damghani MA, Haghdoust AA, et al. Opium and risk of laryngeal cancer. Laryngoscope 2003;113:1939-43.

9 MacLennan R, Da Costa J, Day NE, et al. Risk factors for lung cancer in Singapore Chinese, a population with high female incidence rates. Int J Cancer J Int $d u$ Cancer 1977;20:854-60

10 Masjedi MR, Naghan PA, Taslimi S, et al. Opium could be considered an independent risk factor for lung cancer: a case-control study. Respiration 2013:85:112-8

11 Pourshams A, Khademi H, Malekshah AF, et al. Cohort profile: the Golestan Cohort Study, a prospective study of oesophageal cancer in northern Iran. Int J Epidemiol 2010:39:52-9.

12 Khademi H, Etemadi A, Kamangar F, et al. Verbal autopsy: reliability and validity estimates for causes of death in the Golestan Cohort Study in Iran. PLOS ONE 2010;5:e11183

13 Abnet CC, Saadatian-Elahi M, Pourshams A, et al. Reliability and validity of opiate use self-report in a population at high risk for esophageal cancer in Golestan, Iran. Cancer Epidemiol Biomarkers Prev 2004;13:1068-70.

14 Lyons D, Yazdi I. The carcinogenic potentials of 'opium smoker's tongue'. J Oral Med 1968:24:67-72.

15 Kamangar F, Shakeri R, Malekzadeh R, et al. Opium use: an emerging risk factor for cancer? Lancet Oncol 2014;15:e69-77.

16 Fahmy MS, Sadeghi A, Behmard S. Epidemiologic study of oral cancer in Fars Province, Iran. Community Dent Oral Epidemiol 1983;11:50-8.

17 Nasrollahzadeh D, Kamangar F, Aghcheli K, et al. Opium, tobacco, and alcohol use in relation to oesophageal squamous cell carcinoma in a high-risk area of Iran. Brit J Cancer 2008:98:1857-63.

18 Shakeri R, Kamangar F, Nasrollahzadeh D, et al. Is opium a real risk factor for esophageal cancer or just a methodological artifact? Hospital and neighborhood controls in case-control studies. PLoS ONE 2012;7:e32711.

19 Ghadirian P, Stein GF, Gorodetzky C, et al. Oesophageal cancer studies in the Caspian littoral of Iran: some residual results, including opium use as a risk factor. Int J Cancer J Int du Cancer 1985:35:593-7.

20 Malekzadeh MM, Khademi $\mathrm{H}$, Pourshams $\mathrm{A}$, et al. Opium use and risk of mortality from digestive diseases: a prospective cohort study. Am J Gastroenterol 2013;108:1757-65.

21 Shakeri R, Malekzadeh R, Etemadi A, et al. Opium; an emerging risk factor for gastric adenocarcinoma. Int J Cancer 2013:133:455-61.

22 Naghibzadeh TA, Khanjani N, Yazdi FV, et al. Opium as a risk factor for upper gastrointestinal cancers: a population-based case-control study in Iran. Arch Iran Med 2014;17:2-6.

23 Sadjadi A, Derakhshan MH, Yazdanbod A, et al. Neglected role of hookah and opium in gastric carcinogenesis: a cohort study on risk factors and attributable fractions. Int J Cancer J Int du Cancer 2014;134:181-8.

24 Aliasgari MA, Kaviani A, Gachkar L, et al. Is bladder cancer more common among opium addicts? Urol J 2004;1:253-5.

25 Behmard S, Sadeghi A, Mohareri MR et al. Positive association of opium addiction and cancer of the bladder. Results of urine cytology in 3,500 opium addicts. Acta Cytol 1981;25:142-6.

26 Hosseini SY, Safarinejad MR, Amini E, et al. Opium consumption and risk of bladde cancer: a case-control analysis. Urol Oncol-Semin Ori 2010;28:610-16.

27 Sadeghi A, Behmard S, Vesselinovitch SD. Opium: a potential urinary bladder carcinogen in man. Cancer 1979:43:2315-21. 
28 Karbakhsh M, Dabbagh N, Shabani A, et al. Age at diagnosis in bladder cancer: does opium addiction play a role? Asian Pac J Cancer Prev 2013;14:4723-5.

29 Malaveille C, Friesen M, Camus AM, et al. Mutagens produced by the pyrolysis of opium and its alkaloids as possible risk factors in cancer of the bladder and oesophagus. Carcinogenesis 1982;3:577-85.

30 Hewer T, Rose E, Ghadirian P, et al. Ingested mutagens from opium and tobacco pyrolysis products and cancer of the oesophagus. Lancet 1978;2:494-6.

31 Perry PE, Thomson EJ, Vijayalaxmi, et al. Induction of SCE by opium pyrolysates in $\mathrm{CHO}$ cells and human peripheral blood lymphocytes. Carcinogenesis 1983:4:227-30.

32 Friesen M, O'Neill IK, Malaveille C, et al. Characterization and identification of 6 mutagens in opium pyrolysates implicated in oesophageal cancer in Iran. Mutat Res 1985:150:177-91.

33 Masoudkabir F, Sarrafzadegan N, Eisenberg MJ. Effects of opium consumption on cardiometabolic diseases. Nat Rev Cardiol 2013;10:733-40.
34 Walker PP, Thwaite E, Amin S, et al. The association between heroin inhalation and early onset emphysema. Chest 2015;148:1156-63.

35 Kamangar F, Malekzadeh R, Dawsey SM, et al. Esophageal cancer in Northeastern Iran: a review. Arch Iran Med 2007;10:70-82.

36 Khademi H, Malekzadeh R, Pourshams A, et al. Opium use and mortality in Golestan Cohort Study: prospective cohort study of 50000 adults in Iran. BMJ 2012;344:e2502.

37 Cook-Mozaffari PJ, Azordegan F, Day NE, et al. Oesophageal cancer studies in the Caspian Littoral of Iran: results of a case-control study. $\mathrm{Br} J$ Cancer 1979;39:293-309.

38 Haley KP, Gaddy JA. Helicobacter pylori: genomic insight into the host-pathogen interaction. Int J Genomics 2015;2015.

39 Shakeri R, Kamangar F, Mohamadnejad M, et al. Opium use, cigarette smoking, and alcohol consumption in relation to pancreatic cancer. Medicine (Baltimore) 2016;95:e3922. 